\title{
ESTUARINE ENVIRONMENT STUDIES WITH ISURUS, A REMUS CLASS AUV
}

\author{
Nuno Cruz, Aníbal Matos, Alfredo Martins, Jorge Silva, \\ Domingos Santos, Dmitri Boutov, Diogo Ferreira and F. Lobo Pereira \\ Faculdade de Engenharia da Universidade do Porto and \\ Instituto de Sistemas e Robótica \\ Rua dos Bragas, 4050-123 Porto \\ Portugal
}

\begin{abstract}
In this paper, we address the current developments of the "PISCIS" project. We focus on the experimental results from recent operational missions with an AUV in a estuarine environment. Besides describing mission planning and logistic details, we also present tools for oceanographic data processing and visualization. This will be demonstrated with some examples of collected data.
\end{abstract}

\section{INTRODUCTION}

The Systems and Underwater Technology Lab (LSTS) of the University of Porto has been operating and customizing Isurus, a REMUS class AUV, for the past 2 years.

System capabilities and limitations have been identified and improvements have been incorporated. In particular, all the software has been redesigned, a new navigation system has been implemented and relevant sensors were installed whenever required and viable. Experiments designed to fulfil potential end-user requirements (trying to maximize the collected information about a given process), demonstrated the reliability and the operational effectiveness of this system.

The first operational missions were demonstrated by field tests conducted in the estuary of Minho river in the northern border between Portugal and Spain. During autonomous missions, typically longer than one hour, the vehicle continuously collected CTD and bathymetric data, while navigating on a LBL transponder network.

This paper is organized as follows. In Section II, we briefly describe the "PISCIS" project and the motivation for the use of AUVs in oceanographic missions. In Section III we give a short presentation of the Isurus AUV. Sections IV through VI deal with mission planning, describing the scenario, the mission requirements and the mission editor. We move on, in Section VII, to a discussion on mission logistics, focussing on vehicle tracking and on the steps involved in a typical mission with Isurus. Then, in Section VIII, we show some results of mission data processing. Finally, in Sections IX and $\mathrm{X}$, we discuss the conclusions and future work.

\section{MOTIVATION}

"PISCIS - Prototype of an Integrated System for Coastalwaters Intensive Sampling" is an Eureka R\&D project, supported by the PRAXIS XXI Program. This project envisages the development of a highly operational and lowcost AUV based system for scientific and environmental data collection in the Portuguese coastal waters.
Traditional sampling techniques for underwater observation are generally expensive and do not offer a comprehensive coverage, specially as the requirements of oceanographic and environmental field studies become more and more demanding. In fact, recent advances in ocean sciences and technology have been stressing the importance of efficient observation methods in order to fulfill the requirements of the so-called real-time oceanography $[1,2]$.

AUVs may be efficiently used in a wide range of applications. They were first developed with military applications in mind, namely for mine hunting missions. Later on, scientists realized their true potential and started to use them as mobile sensors, taking samples in difficult scenarios at a reasonable cost. Currently, there is an increasing worldwide effort concerning the design and implementation of AUVs. This trend will result in the availability of fully operational vehicles that will make it possible to envisage the operation of AUVs in more complex applicational scenarios.

The development of a system such as the one devised has much to gain from the interaction with demanding potential end-users. The information gathered from the requirements of realistic missions is proving to be invaluable in the overall system design. The resulting system will emerge from a tradeoff between this top-down perspective and a bottom-up approach driven by the constraints imposed by state-of-the-art technologies.

\section{THE ISURUS AUV}

Isurus (Fig. 1) is a REMUS (Remote Environment Measuring UnitS) class AUV, acquired from the Woods Hole Oceanographic Institution, MA, USA, in 1997. REMUS vehicles are low cost, lightweight AUVs specially designed for coastal waters monitoring (maximum depth of 200m) [3]. The reduced weight and dimensions makes them extremely easy to handle, not requiring special equipment for launching and recovering, such as winching systems.

Isurus has a diameter of $20 \mathrm{~cm}$ and is about 1.5 meters long, weighting about $30 \mathrm{~kg}$ in air. This vehicle can cruise at a maximum forward speed of 4 knots, however the best energy efficiency is achieved at about 2 knots. At this velocity, the energy provided by the rechargeable lead acid batteries last for over 10 hours (i.e., over 20 nautical miles). We are currently evaluating other options for the batteries, based on technologies that allow for higher energy densities, namely Nickel Metal Hydride and Lithium-Ion. 


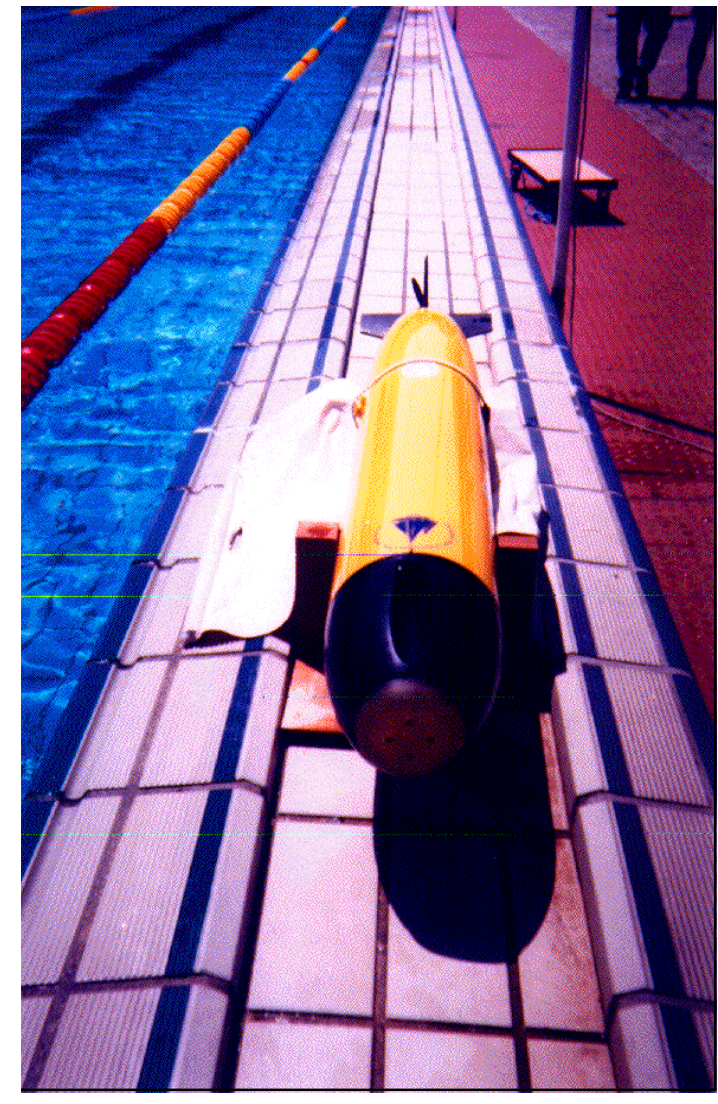

Figure 1 - The Isurus AUV

REMUS original acoustic navigation system could be used in either LBL or USBL mode. A dedicated DSP processed the signals received by the hydrophones. We have implemented a new navigation system, which only operates in LBL mode, but where the DSP based signal detection was replaced by analog filters tuned to the frequencies of the signals emitted by the transponders. An algorithm that fuses range measurements with dead-reckoning information was also implemented in the on-board computer.

Although small in size, it is possible to accommodate a wide range of oceanographic sensors, according to mission objectives. For the missions described in this paper, we have integrated a CTD (from Ocean Sensors, CA, USA) and an altimeter (from Imagenex, Canada) in a specially designed nose extension.

A wide range of sensors can be easily integrated on these vehicles. Successful tests have been performed in the past with sidescan sonars, ADCP (Acoustic Doppler Current Profiler), and Optical BackScatter.

\section{MISSION SCENARIO}

Cooperation with oceanographers and marine biologists from Porto University were instrumental to produce a set of application scenarios in which AUV based systems are clearly advantageous compared to alternative existing methods [4]. One of these scenarios consists in the use of AUVs for the identification of relevant factors affecting the eel recruitment process in estuarine environments. Aspects of the dynamics of the coastal ocean and the estuary, such as horizontal and vertical circulation are believed to play an important role.
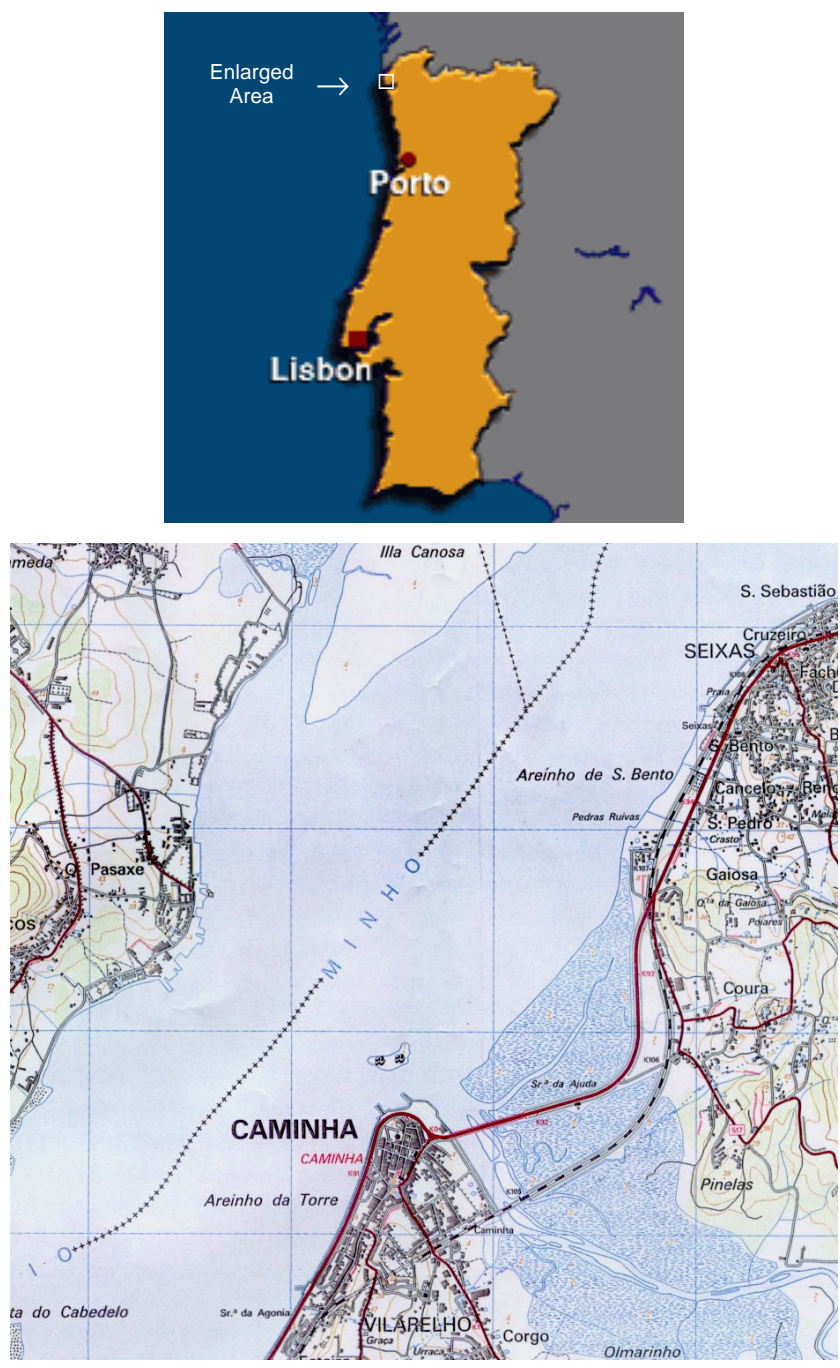

Figure 2 - Mission scenario overview (4km x 4km)

The scenario chosen for the first operational missions was the estuary of Minho river in the northern border between Portugal and Spain (Fig. 2), for 3 main reasons:

- Logistics: A marine station situated in this estuary provided the facilities required for field testing and development, including a small boat to support the missions.

- Previous knowledge: This river has been the subject of study and active research during the last 15 years. This has provided for some knowledge on the operational scenario, namely currents, depth profiles, tides, etc.

- Scientific relevance: Several studies, including $2 \mathrm{PhD}$ thesis, have been recently conducted in this environment, particularly about the eel population. The hydrological aspects have been less studied, but it seems that the AUV measurements can provide information for a better understanding on the evolving ecological processes. 
The estuary is about $5 \mathrm{~km}$ long, with a maximum width of about $2 \mathrm{~km}$ and a very narrow mouth. Due to the specific characteristics of this estuarine region, the strong interaction between fresh and salt water causes circulation patterns with great variability in the values of temperature and salinity. Previous data also showed that typical currents can be over 1 knot, particularly during tidal changes.

As far as bathymetry is concerned, there are a few old charts, but the riverbed is continuously changing due to the strong dynamics. Typical depth values indicate depths around $3 / 5$ meters, although there is a deeper channel well known to fishermen.

\section{MISSION DESIGN}

Estuarine circulation is usually very complex and horizontal sampling with the AUV at different depths may show circulation patterns very difficult to observe with any other technique. In order to achieve this, several cross sections (about 500 meters apart) should be performed, with the depth varying from the surface to the bottom. This should be repeated for different tides. Data to be collected included temperature and salinity. Other interesting data would be Dissolved Oxygen, but this sensor was not yet installed when the missions started. Later on, atmospheric parameters such as air temperature, luminosity and wind should also be analyzed.

The availability of an onboard altimeter allowed to test another type of mission: autonomous bathymetry. The idea was to evaluate the possibility of using the AUV to map the bottom of the river and construct its 3D map. If viable, the vehicle could be deployed in consecutive sections of the river, in order to build an updated map of the bottom. Besides demonstrating the vehicle as a tool for underwater mapping, these charts would also allow for a safer navigation. In the longer term, they may also be used to detect changes in the topography, map the ferry-boat channel, help in the construction of oceanographic models and the construction of 3D virtual environments for mission simulation.

\section{MISSION EDITING}

An AUV mission starts with the vehicle programming, by editing a mission file with the objectives that need to be successively accomplished.

In the original implementation, a text file would have to be edited, which would result in a hard to debug file. Furthermore, the new QNX software architecture allows for the design of new mission objectives, as required, for example, in adaptive sampling missions (in which case, the mission goals may not be fully defined a priori).

For these reasons, we have redesigned the mission structure and we have developed a graphic mission editor to facilitate mission edition [6]. The new set of objectives encompasses all the previous ones and includes a few others, where the most important changes are related to adaptive sampling objectives. A different approach was taken as far as vehicle protection modes are concerned. We now have a set of protection modes for each objective. Each mode may be enabled or disabled, and for each enabled protection, there is an emergency behavior associated (i.e., tells the vehicle what to do if the protection is triggered).

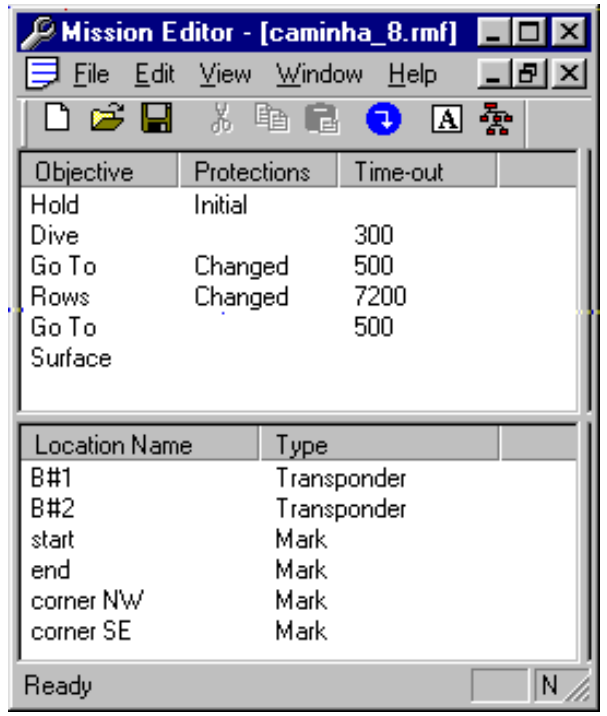

Figure 3 - A typical mission window

Figure 3 shows a simple mission window, where the vehicle is sent to a starting point, from where it swims in a lawnmower pattern ("Rows"). At the end, it is sent to the end point, where it surfaces to be recovered.

By selecting an objective, the user may edit the specific details. Figure 4 shows details of the "Rows" objective. In this case, the rows are 500 meters long and the objective would be completed after 5 consecutive rows, with 50 meters spacing between them.

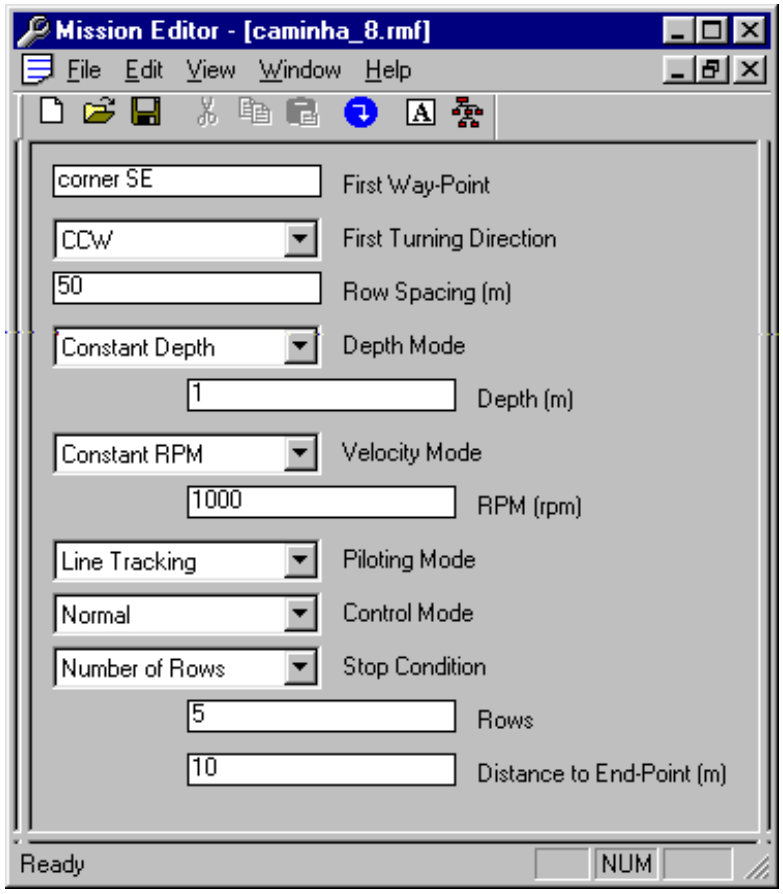

Figure 4 - Parameters for the "Rows" objective 


\section{LOGISTICS}

\section{A. The PAL System}

There is a significant risk of loosing an AUV while performing autonomous operations. However, Isurus has an automatic responder that sends a specific acoustic signal whenever an interrogation is received. This systems has a backup battery that keeps it working in the event of a failure in the main power system.

When the first field trials started, we developed PAL (Portable Acoustic Locator), a simple device with 2 purposes: to be able to generate a series of acoustic signals, interpreted by the vehicle as an abort command, and to measure distance to the vehicle by measuring the time elapsed between the interrogation signal and the vehicle's response.

PAL has been quite helpful during operations and it has been successively upgraded to measure ranges to different devices. Now, it is also possible to use it to know the position of the vehicle with respect to the baseline. We integrated new filters for the transponder receiver frequencies, so that it is possible to detect when the vehicle interrogates each of the transponders. Since the vehicle is consecutively switching from one transponder to the other, we can measure the time between interrogations and, so, determine the range to each of the transponders. This is only possible with the new LBL navigation system [5] and the new vehicle software [6], implemented on the QNX real time operating system. These two systems ensure that the vehicle switches to the other transponder immediately after detecting a response (the uncertainty in this switching time is kept below $1 \mathrm{~ms}$ ). The best feature about this mode of operation is that it is completely passive, that is, it is possible to know the vehicle position just by listening to its normal operation. Furthermore, it does not depend on the location of the PAL transducer, since it only deals with time differences.

\section{B. A Typical Mission}

During this mission, the AUV would be navigating on an LBL transponder network. The estuarine scenario is characterised by having poor acoustics, so we had to increase the threshold levels for acoustic detection, reducing the maximum length allowed for the baseline. For this mission, we only used 2 transponders, defining a baseline not larger than 1 $\mathrm{km}$.

It is difficult to work on the boat, even in a relatively calm day, so we tried to have the mission ready before leaving the marina. The only information missing before leaving was the exact transponder location.

After editing the mission objectives in the laptop, the final diagnostics were made, during which the various subsystems were verified (Figure 5). The pressure sensor was reset to compensate for changes in the atmospheric pressure and the compass was calibrated to compensate for local variation in the magnetic field.

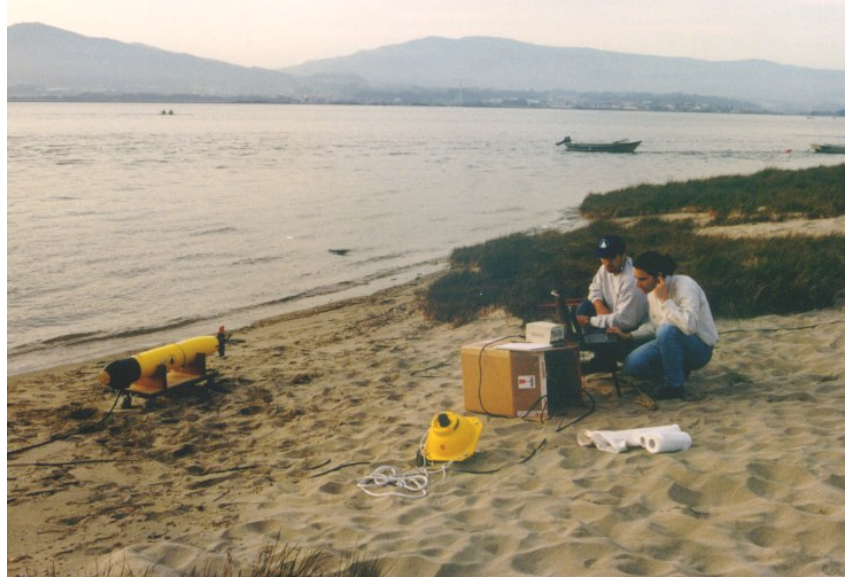

Figure 5 - Final diagnostics by the riverside

Isurus was then placed on the boat, along with the few things required for mission support: 2 transponders with anchors and surface expressions, GPS receiver, KVH Datascope, PAL, and a laptop.

The first navigation transponder was deployed with the aid of a portable GPS receiver. Since there are no DGPS reference stations in the region, the position error was about 50 meters. We then used a KVH Datascope to provide precision bearings to natural references (whose positions were already stored in the laptop). Triangulation of these bearings allowed for an overall accuracy of about 10 to 20 meters.

For the second transponder, we went to the indicative GPS position and repeated the above process. However, at this time, we were also able to use Datascope to determine the bearing from the first transponder, and, with PAL, we measured range between both transponders. This way, we had enough information for a precise positioning (in fact, a lot better than required for this mission).

With the determination of the exact transponder location, the mission file was completed and transferred to the vehicle. PAL was then connected to the laptop serial port and the transducer was placed in the water. When the laptop tracking program started running, the vehicle was ready to start the mission.

At the end of the mission, the vehicle surfaced and stopped pinging the transponders, so the tracking program could not locate the vehicle any longer. We then moved the boat to the last known position and use the range utility (with PAL) to find it.

The mission operations ended with the recovery of the transponders (PAL was also used when they were hard to find) and the download of all the collected information.

\section{MISSION DATA PROCESSING}

There are two data sets collected during a typical mission. The first set is related to the vehicle internal data, which is only analyzed in the lab. This allows to evaluate the performance of the various subsystems (navigation, control, power consumption, etc) and provide useful information for 
potential improvements. The other data set comprise all the information from the oceanographic sensors.

The oceanographic data requires time and position information. This is done by post-mission filtering of the navigation information, in order to correct for eventual positioning errors that may have occurred during the mission.

Measurements can be delivered to the end-users as raw data or they can be processed to allow for a rapid validation. In order to reduce this validation time, and also to help us troubleshooting any vehicle problem, we designed a Matlab application for data processing and visualization.

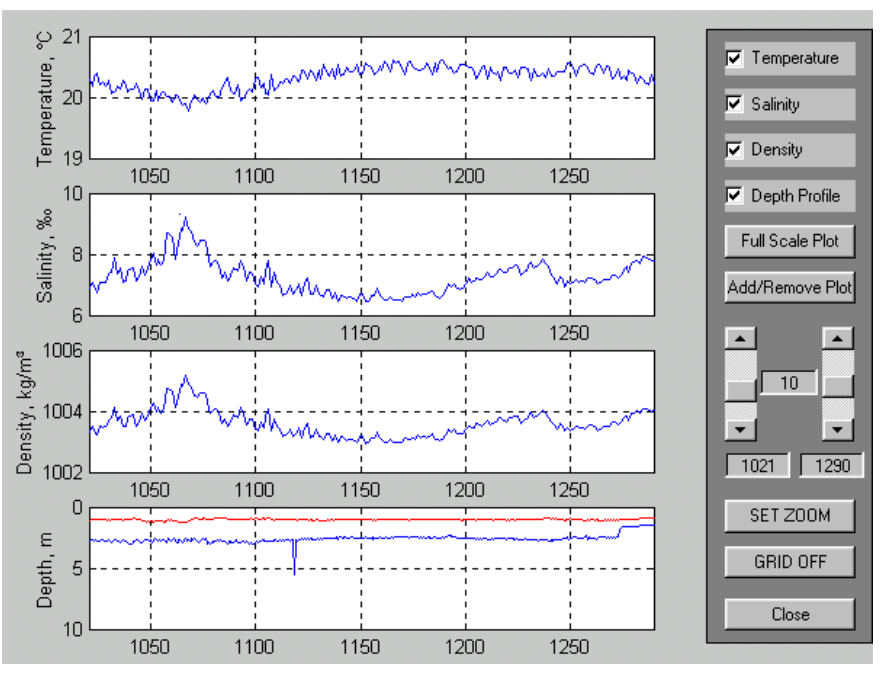

Figure 6 - Matlab application window with ocean data

Once the program starts, mission data needs to be loaded (by clicking a "Load Data" button). The user can then select the type of information to plot: ocean data, vehicle data, or custom. This last option allows to select any variables and is specially useful for troubleshooting. Figure 6 shows a plot of the ocean data for a part of the mission.

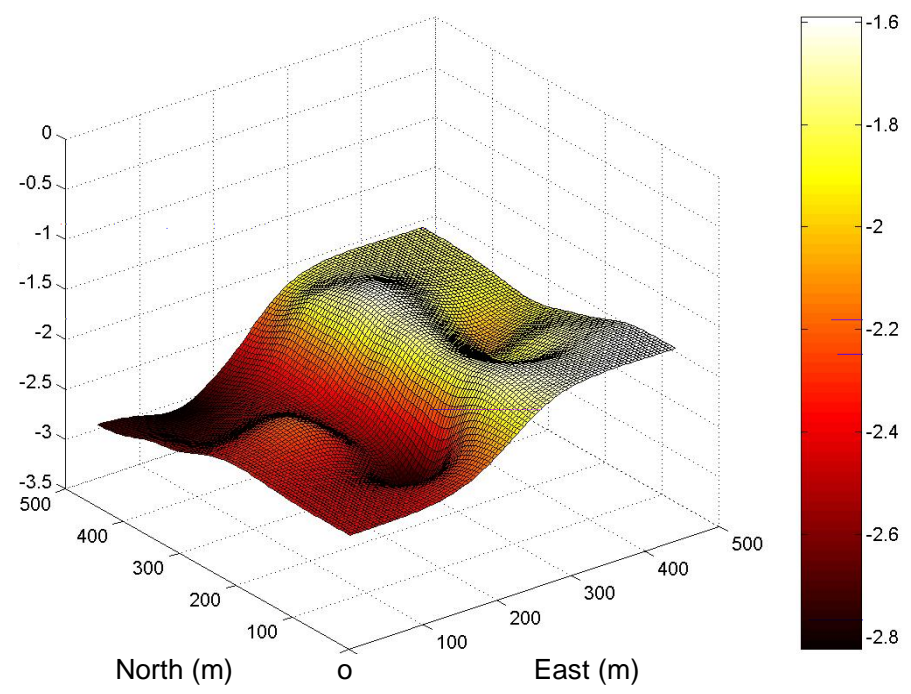

Figure 7 - Bathymetry of the sampled area
Oceanographic data can also be displayed in 2D or 3D maps, after filtering. Figure 7 shows the result of filtering bathymetry data and interpolating where there was no data available. It should be pointed out that in this particular mission, the row spacing was 50 meters, which only allows for a low-pass interpolation. This is the reason why the shape of the bottom is smoother that in reality. In order to obtain a more accurate description of the bottom, we would need to process data from a mission with less row-spacing.

In Figure 8, we can see temperature values for the same region as above, at 1 meter of depth. In this case, we plotted isotherms and a temperature distribution map.

In both figures, the plot reference $(0,0)$ corresponds to coordinates (N 4153'33',0; W 849'53”,4).

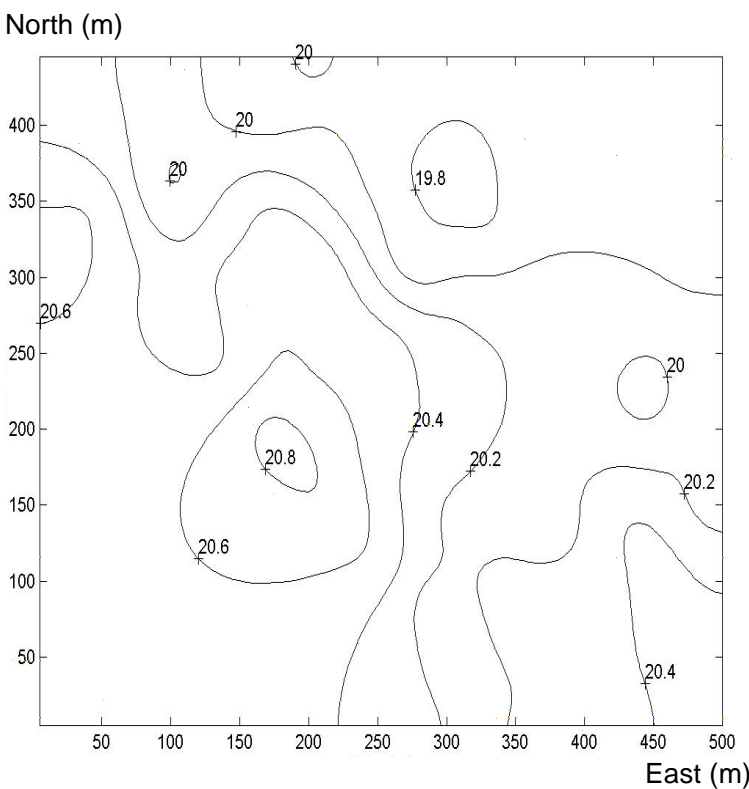

North $(m)$

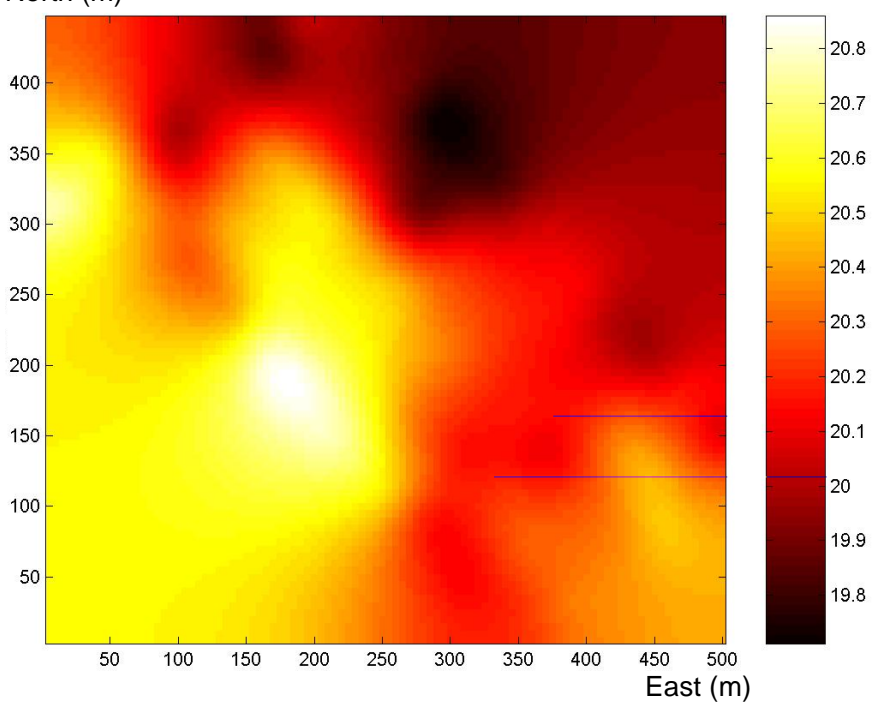

Figure 8 - Isotherms and temperature map 


\section{CONCLUSIONS}

The autonomous operation of the AUV, collecting relevant CTD and bathymetry data during over an hour with no operator intervention, was a pioneer experience in Portugal. In fact, the Isurus AUV succeeded in performing the programmed missions, even in adverse operational conditions, both weather-related (some of the sections were conducted with heavy rain and strong winds) and underwater environment (very shallow water).

The estuary of the Minho river is a challenging environment for AUV operations, since it is very shallow, with typical depths varying from 2 to 5 meters, and highly significant water currents during tide changes.

The high variability of the environment and also the need to collect the samples rapidly, before tide changing, contribute for the importance of the AUV as the most efficient way of mapping the underwater environment, as could be realized by the quasi-synoptic image of the river characteristics.

The shallowness of the environment and the variability of the bottom topography pose serious problems for depth control. In fact, the errors typically accepted as irrelevant when operating in tens or hundreds of meters, become very important in underwater environments as shallow as this one. At the end, we achieved a standard deviation of $7 \mathrm{~cm}$ from the required depth, which is a good measure of performance for the depth controllers.

The acoustic conditions at this scenario are about the worst than can be found in an underwater environment. Acoustic navigation has to deal with multipath for the sound waves, which means that the distance between the vehicle and each of the transponders had to be reduced. Furthermore, the high variability of the hydrological values significantly affects the sound speed, also increasing positioning errors. In this case, we had to operate with baselines about $1 \mathrm{~km}$, in order to have reliable navigation. Even at this small distance, the vehicle would often experience difficulties in detecting transponder replies, probably due to channeling of the acoustic waves. However, overall vehicle navigation proved to be smooth.

The whole system is becoming more complete, but remains highly operational. The estuarine environment was first used as a testbed for new subsystems test and integration and most of the laboratory equipment required for development was easily transported to the station. The small boat available for operational support was sufficient for the estuarine mission. Data visualization immediately after mission completion is very useful to validate mission.

Operational missions have been facilitated by the PAL system, which has provided for a high degree of confidence when the vehicle is performing long autonomous missions.

\section{FUTURE DEVELOPMENT}

We are now in the process of installing an underwater video camera in the vehicle. This will be particularly important for an environmental monitoring mission to be performed in the near future. This mission will take place in a sewage outfall region off the Portuguese coast, where regular inspection is required, comprising in situ water analysis and visual inspection of the underwater pipeline. The vehicle will travel about $2 \mathrm{~km}$ while maintaining the pipeline in the camera field of view. Later on, water monitoring sensors will be added in order to accomplish the whole monitoring plan.

Other planned missions include precise bathymetry of a large river dam (to study bottom erosion) and visual inspection of artificial reefs installed off the Portuguese coast.

As far as software is concerned, the mission editor is being upgraded, in order to include a tool for mission validation. Even though the objectives are already individually validated, the idea is to test overall mission integrity.

The fact that the position of PAL transducer is irrelevant to track the vehicle (as long as it is kept within maximum range), allows for the implementation of a new system for remote tracking. The idea is to place a passive device in the operation area and transmit data to the shore station through a radio link.

The laboratory will maintain the present strategy for the longer term. New partners and end users will be sought. New sensors will be added as required by their requirements.

These activities constitute the basis to achieve the ultimate goals of the project, namely performing autonomous environmental monitoring missions off the Portuguese coast with single and multiple vehicles, performing autonomous underwater docking and, finally, contribute for the establishment of a permanent underwater observatory.

\section{REFERENCES}

[1] T. Curtin, J. Bellingham, J. Catipovic and D. Webb. Autonomous Oceanographic Sampling Networks. In Oceanography, Vol. 6, No. 3, 1993, pp. 86-94.

[2] F. Lobo Pereira, J. Borges de Sousa, C. Gil Martins, E. Pereira da Silva. AUV System Requirements for Coastal Oceanography. In Proceedings of the IEEE AUV'96 Conference, Monterey, CA, USA, June 1996, 399-406.

[3] C. Alt, B. Allen, T. Austin and R. Stokey. Remote Environmental Measuring Units. In Proceedings of the Autonomous Underwater Vehicle's 94 Conference, Cambridge, MA, USA, July 1994, pp. 13-19.

[4] J. Borges de Sousa, N. Cruz, A. Matos and F. Lobo Pereira. Multiple AUVs for Coastal Oceanography. In Proceedings of the MTS/IEEE Oceans'97 Conference, Halifax, Nova Scotia, Canada, October 1997.

[5] A. Matos, N. Cruz and F. Lobo Pereira. Development and Implementation of a Low-Cost LBL Navigation System for an AUV. To apper in the Proceedings of the MTS/IEEE Oceans'99 Conference, Seatle, WA, USA, September 1999.

[6] J. Silva, A. Martins and F. Lobo Pereira. A Reconfigurable Mission Control System for Underwater Vehicles. To apper in the Proceedings of the MTS/IEEE Oceans'99 Conference, Seatle, WA, USA, September 1999. 